Théologiques

Théologiques

\title{
Misères et grandeurs de la bioéthique
}

\section{Guy Durand}

Volume 7, numéro 1, printemps 1999

Les trente ans de la bioéthique

URI : https://id.erudit.org/iderudit/024973ar

DOI : https://doi.org/10.7202/024973ar

Aller au sommaire du numéro

\section{Éditeur(s)}

Faculté de théologie de l'Université de Montréal

ISSN

1188-7109 (imprimé)

1492-1413 (numérique)

Découvrir la revue

\section{Citer cet article}

Durand, G. (1999). Misères et grandeurs de la bioéthique. Théologiques, 7(1), 51-73. https://doi.org/10.7202/024973ar

\section{Résumé de l'article}

Cet article jette un regard critique sur la bioéthique, tant sur ses aspects positifs que sur des aspects jugés plus controversés. De l'utilisation du terme lui-même en passant par le contenu, la nature et les fondements de la bioéthique, on cherche ici à dégager, à travers de multiples propositions avancées par les chercheurs, des avenues susceptibles de tracer la voie pour la suite des recherches dans ce secteur en pleine expansion. d'utilisation que vous pouvez consulter en ligne.

https://apropos.erudit.org/fr/usagers/politique-dutilisation/ 


\title{
Misères et grandeurs de la bioéthique
}

\author{
Guy DURAND \\ Professeur émérite \\ Faculté de théologie \\ Université de Montréal
}

J'ai hésité entre deux titres: Misères et grandeurs de la bioéthique, qui indique bien le sens de l'exposé* ; Controverses et malentendus, qui désigne mieux son contenu. De toute manière, je veux présenter un certain nombre de malentendus ou de controverses qui marquent la bioéthique actuelle et qui déterminent, en deçà de sa grandeur, ses misères et ses travers.

Ce faisant, il m'arrivera de décrire la situation actuelle, de dégager les enjeux en cause, de signaler où je me situe moi-même. Voulant respecter les limites d'un article, j'ai forcément laissé de côté plusieurs nuances. On pourra trouver plus d'explications dans un volume à paraître en 1999 .

\section{Ambiguïtés du mot}

Le mot «bioéthique» est ambigu. Cette ambiguïté tient à plusieurs éléments. Commençons par les plus faciles.

1) La première ambiguïté vient du préfixe bio. Le mot désigne-til une éthique biologique, c'est-à-dire une éthique élaborée à partir de la biologie 1 ? Presque tous les éthiciens diront qu'il y a là une

\footnotetext{
* Ce texte fait suite à une conférence donnée le 20 nov. 1998, à l'U. de M. dans le cadre de la journée des diplômés du DESS en bioéthique. 1 Voir l'excellent débat entre A. COMTE-SPONVILLE et L. FERRY, La sagesse des modernes. Dix questions pour notre temps. Paris, Robert Laffont,
} 
confusion d'ordres. L'éthique ne se déduit ou ne s'induit pas de la biologie : on ne passe pas automatiquement de la constatation des faits à l'affirmation des valeurs. Ce qui existe (les mœurs, le techniquement possible, le légal) n'est pas nécessairement éthique. Même si les faits, les connaissances scientifiques, doivent toujours nous laisser interrogateurs, les lois biologiques ne déterminent pas l'éthique.

Par ailleurs, s'agit-il d'une éthique du vivant, une éthique de la vie, de la survie de l'espèce? Cela correspondrait à la perception qu'en avait le créateur du mot en 1970, le cancérologue américain Van Rensselaer Potter ${ }^{2}$ lequel incluait dans la bioéthique ce qu'on appelle aujourd'hui l'éthique environnementale. Cette notion correspondrait aussi à l'étymologie (bios) et à la perception de certains interlocuteurs. Cependant, l'histoire récente notamment sous l'influence du Kennedy Institute of Ethics ${ }^{3}$ l'a plutôt restreinte au domaine biomédical i.e. au domaine de la vie humaine: naissance, santé, maladie, mort. Le champ est déjà très vaste, l'horizon des connaissances scientifiques déjà presque trop grand. L'élargir davantage, à mon avis, n'aide pas à la compréhension. Et il y a lieu de se

1998, pp. 71-135. Voir J.-P. CHANGEUX (dir.), Fondements naturels de l'éthique. Paris, Odile Jacob, 1991, 334 p.

2 Van Rensselaer POTTER, "Bioethics, the Science of Survival" dans Perspectives in Biology and Medicine 14 (1970) 127-153; Idem, Bioethics: Bridge to the Future. Englewood Cliffs, New Jersey, Prentice-Hall Inc., 1971, p. 205 (préface et chap. 1 intitulé Bioethics, the Science of Survival). La pensée de Potter (et son héritage) est bien présentée par H. DOUCET dans $A u$ pays de la bioéthique. L'éthique biomédicale aux États-Unis. Genève, Labor et Fides, 1996, pp. 36-43.

3 La paternité respective de Potter et de Hellegers est très bien présentée par W.T. REICH, "How Bioethics Got Its Name» dans Special Supplement, Hastings Center Report 23 (nov.-déc. 1993) 5-7; Idem, "The Word 'bioethics' : its Birth and the Legacies of Those Who Shaped Its Meaning " dans Kennedy Institute of Ethics Journal 4 (déc. 1994) 319-335. Elle est bien reconnue aussi par Van Rensselaer POTTER, "Aldo Leopold's Land Ethics Revisited: Two Kinds of Bioethics" dans Perspectives in Biology and Medicine 30 (hiver 1987) 157-169. 
méfier de la tentation de chercher à inclure dans un mot populaire toute la réflexion éthique. L'éthique environnementale est importante, certes, elle constitue un chapitre important de l'éthique mais il n'y a pas lieu, je crois, de l'inclure dans la bioéthique.

2) La deuxième ambiguilté vient du mot éthique. D'une part, le mot s'est imposé, par opposition à celui de morale, qui faisait conser vateur, fermé, parfois religieux ${ }^{4}$. À l'inverse, l'éthique connotait un questionnement neuf, ouvert, prospectif, voire interdisciplinaire. Il était prometteur d'une réflexion fondamentale, exigeante. D'autre part, depuis les années 70 , le mot a été employé à toutes les sauces. Plusieurs professions, plusieurs institutions ont voulu se donner des codes d'éthique, qui sont loin de ce qu'on entendait jadis par l'exigence éthique. Au point où un philosophe comme Alain Etchegoyen a publié un livre au titre évocateur: "La valse des éthiques 5 ". D'autres observateurs ont suggéré qu'on a privilégié volontairement le terme éthique pour esquiver les véritables exigences morales ${ }^{6}$.

Toujours est-il que le mot admet des significations différentes selon les auteurs, significations souvent difficiles à saisir parce que liées chacune à un système philosophique particulier et, par conséquent, difficiles à comparer entre elles et à sérier. Ce qui met certains lecteurs devant un éventail paralysant.

Pour ma part, j'emploie assez indifféremment un mot pour l'autre, tel que l'étymologie, l'histoire et un certain usage philosophique le

4 Dévalorisation, négativité attestées par des auteurs de divers pays. Exemples : F. DUMONT, Situation et avenir du catholicisme québécois : entre le temple et l'exil. Montréal, Leméac, 1982, tome 2, p. 33; D. CALlAHAN, "L'éthique biomédicale aujourd'hui " dans Éthique et biologie (Cahiers STS), 11 (1986) 46-48; J. BERNARD, "Évolution de la bioéthique " dans Médecine/Sciences 9/2 (nov. 1986) 480; Idem dans CCNE, Rapport 1984, p. 195.

5 A. Etchegoyen, La valse des éthiques, Paris, Éd. François Bourin, 1991.

6 J. LEJEUNE, dans Revue des écrivains catholiques 21 (mars 1985). 
justifient, tout en étant conscient de certaines différences et de certaines nuances reliées aux mots?

3) Une troisième ambiguïté vient de l'origine américaine ou mieux états-unienne du mot. Venant des États-Unis, le mot séduit ou irrite: c'est selon. Les deux attitudes existent d'ailleurs en Europe comme au Québec. Parfois, on copie les Américains presque servilement. D'autres fois, on s'en méfie et on les critique exagérément sans tenir compte de l'histoire et du contexte général.

Plus profondément, la bioéthique est souvent identifiée à la conception qu'on s'en est faite aux États-Unis dans les années 70, notamment autour de The National Commission for the Protection of Human Subjects of Biomedical and Behavioral Research (1974-78) et autour du Kennedy Institute of Ethics, fondé en 1971. Hubert Doucet, dans un livre excellent intitulé $A u$ pays de la bioéthique, décrit très bien cette conception, appelée conception canonique, ou encore, en anglais, principlism, centrée sur trois ou quatre principes (autonomie, non-malfaisance, bienfaisance, justice ${ }^{8}$ ). Hubert Doucet parle d'une conception pragmatique, peu intéressée aux débats théoriques ou aux fondements, mais centrée sur la résolution de problèmes et la prise de décision et où les quatre principes jouent un peu comme des mantra ${ }^{9}$. Plusieurs auteurs européens critiquent la bioéthique en s'attaquant de fait à ce seul courant pragmatique ${ }^{10}$.

7 Voir G. DURAND, La bioéthique: nature, principes, enjeux. Paris, Cerf; Montréal, Fides (Bref), 1989 et 1997, pp. 11-21.

8 H. DOUCET, Au pays de la bioéthique, pp. 63-100.

9 Dans un article critique de la bioéthique, Danner Clouser et Bernard Gert commencent ainsi leur texte: "Dans tout le pays, des foules de convertis à la conscience bioéthique font entendre leur mantra 'bienfaisance... autonomie... justice...' Cette incantation rituelle pour résoudre les dilemmes bioéthiques est à l'origine de notre recherche." Voir "A Critique of Principlism", dans The Journal of Medicine and Philosophy 15 (1990) 219.

10 Voir J.-M. THÉVOZ, "Place de la théologie dans le débat bioéthique » dans Le Supplément 178 (1991) 127 et ss.; D. MÜLLER, «Situation 
Cela dit, malgré ces ambiguités, le mot reste utile. Il n'est pas moins heureux que ses concurrents. Et il décrit très bien ce qu'on veut dire. Il est de plus en plus employé dans tous les milieux, tant au plan national qu'internationall1.

\section{Querelles sur la nature de la bioéthique}

Dans son livre sur la bioéthique aux États-Unis, Hubert Doucet analyse les divers courants de pensée qui s'y expriment, se complémentent et/ou s'affrontent : le courant canonique d'abord, appelé le principlism; ensuite certaines ré-interprétations de ce courant, centrées sur la relation professionnel/patient ou sur l'aspect social des problèmes; enfin des voies différentes : l'éthique de la vertu, le courant féministe, la casuistique, la narrativité ${ }^{12}$. Ces courants ont une influence certaine sur la réflexion et la pratique bioéthiques.

Cependant mon propos ici est différent. Au-delà de ces courants, les traversant en quelque sorte, se pose la question de la nature de la bioéthique. Qu'est-ce que la bioéthique? La bioéthique est-elle de l'éthique? Est-elle une réflexion d'ordre éthique? À ce propos existent plusieurs controverses. J'en retiens deux : la bioéthique estelle de l'éthique? Est-elle de l'éthique appliquée?

\subsection{L'ordre éthique}

$\grave{A}$ mon avis, il est instructif de distinguer quatre courants, quatre tendances, qui s'expriment dans autant de définitions et qui cachent quatre façons de voir la nature de la bioéthique. Il s'agit de ten-

et statut de l'éthique médicale » dans Ethik in der Schweiz, Theophil 2 (1996) 169-175.

11 Voir les publications du Comité consultatif national d'éthique (CCNE) en France, les lois de la bioéthique élaborées par le Conseil de l'Europe, le nom du Comité de l'ONU sur les questions d'éthique biomédicales.

12 H. DOUCET, Au pays de la bioéthique, pp. 101-156. 
dances, un peu comme de modèles théoriques, que je durcis légèrement pour mieux faire saisir les divergences et donc les enjeux ${ }^{13 .}$

1) Certains, en effet, voient la bioéthique comme un simple cadre de réflexion et de recherche multidisciplinaire sur les enjeux des développements technico-médicaux. La bioéthique ne viserait qu'à mettre ensemble, par exemple, dans des groupes d'étude ou des congrès, des spécialistes de diverses disciplines. La bioéthique désignerait une sorte de forum, de préoccupation commune, où l'apport de chacun resterait plus ou moins juxtaposé. La plus belle image de cette conception de la bioéthique est le panel rassemblant des intervenants de diverses disciplines où chacun (y compris l'éthicien) expose son point de vue, mais où il n'y a presque pas de temps consacré à l'échange et la discussion. Beaucoup de livres de bioéthique sont aussi construits sur ce modèle: un chapitre d'ordre biologique, un autre d'ordre psychosocial, un autre d'ordre juridique et un dernier (écrit avant ou en même temps) sur l'éthique. La caractéristique principale y est la juxtaposition des apports.

La définition de la bioéthique comme champ de réflexion risque de donner dans ce courant. Les biotechnologies médicales ou, en d'autres termes, les questions de vie, mort, santé, maladie, constituent incontestablement un champ d'étude et de réflexion multidisciplinaire. Le biologiste, le biochimiste, etc., y ont quelque chose à dire, à faire. Le travailleur social, le juriste, l'anthropologue, eux aussi. Mais font-ils de la bioéthique quand ils se limitent à réfléchir selon leur point de vue disciplinaire ou à mettre vaguement ensemble leur apport disciplinaire? Nous y reviendrons.

2) En allant plus loin, d'autres voient dans la bioéthique une question de méthode ou de technique de résolution de problèmes et de prise de décision, la bioéthique désignerait une approche nouvelle, interdisciplinaire, pragmatique; ou encore une méthode d'analyse interdisciplinaire et pragmatique encore une fois, mais aussi situationnelle,

13 On trouvera une première version de cette typologie dans mon livre La bioéthique: nature, principes, enjeux, déjà cité. S'agissant de modèles théoriques, il est difficile de mettre des noms sans caricaturer les points de vue. 
inductive, holiste. La bioéthique viserait à mettre au point des grilles d'analyse de cas complexes, des processus de prise de décision dans des situations où plusieurs intervenants sont impliqués. Elle serait centrée sur les analyses de cas et la solution de dilemmes moraux, notamment par l'analyse des avantages et inconvénients, «des coûts et bénéfices ». Elle est orientée vers l'action.

Elle s'apparente alors au "case studies " que l'on retrouve beaucoup en sciences infirmières et en travail social, ou à la " formation par problèmes" développée dans plusieurs facultés de médecine en Amérique du Nord. Elle s'en distingue surtout en ce qu'elle vise à expliciter (mettre à jour, clarifier, analyser) les aspects éthiques ou les valeurs impliquées.

Ce courant peut relever d'une attitude très pratique. Il faut trouver une solution à des problèmes concrets, urgents, auquel cas il n'y a rien à redire. Il peut se rattacher aussi à ce qu'on appelle en philosophie une éthique procédurale. Conscient qu'il n'y a pas unanimité dans nos sociétés sur les questions de fond, souvent même convaincu qu'il n'y a aucune vérité absolue (aucune vérité métaphysique), on se réfugie dans le processus de discussion. Il n'y a de vérité que celle qui sourd d'un groupe qui discute selon des règles adéquates.

On aura compris que cette perspective élude les questions de fond. Elle se limite aussi aux seules situations où il y a des dilemmes, des conflits.

3) On peut dégager une troisième conception où l'on conçoit la bioéthique comme un processus de régulation sociale. Cette tendance, elle aussi d'ordre pragmatique, est dominée par la question du consensus et du compromis social. Ses tenants prennent pour acquis que, dans un monde pluraliste, le consensus sur les questions de fond (nature de fœtus, acceptabilité du clonage, moralité de l'euthanasie active, etc.) est impossible. Aussi ont-ils déplacé leurs efforts sur la recherche de compromis pratiques, opérationnels. La recherche d'un consensus porte d'abord sur les règles du jeu en permettant aux diverses options éthiques d'exister en brimant le moins possible les consciences; il porte ensuite, si possible, sur le contenu, sur les valeurs elles-mêmes. Cette tendance vise alors à dégager une sorte d'éthique consensuelle, entérinant les valeurs et/ou posant les condi- 
tions au-delà desquelles une forte majorité de citoyens ne seraient pas d'accord. On vise souvent à dégager «le plus grand commun dénominateur ", mais qui ne constitue toujours qu'une éthique minimale.

Processus de régulation sociale, cette tendance se rapproche considérablement du droit, notamment en autant qu'elle tend à gommer les différences idéologiques entre citoyens et invite à se concentrer sur la recherche de compromis social. Elle s'en distingue en ce qu'elle ne cherche pas tant à concevoir des lois à imposer à la société (encore qu'elle peut aller jusque là) qu'à établir des normes institutionnelles, corporatives, qui sont davantage des repères, des lignes directrices, des orientations. La bioéthique ainsi conçue se distingue aussi du droit en ce qu'elle porte sur des objets plus étendus, par exemple des pratiques quotidiennes ou des possibilités technoscientifiques nouvelles sur lesquelles il n'y a pas de loi et sur lesquelles il n'est peut-être pas opportun de faire des lois, du moins pour le moment.

4) La quatrième tendance regroupe des auteurs qui veulent mettre davantage en relief la perspective éthique du mot et de la réflexion. Aussi ses tenants insistent-ils sur les diverses dimensions de l'éthique (recherche personnelle approfondie; analyse des enjeux éthiques des questions ou des cas; examen critique des principes, des valeurs et des postulats fondamentaux; effort de systématisation et de cohérence), cherchant à dégager un idéal, éventuellement très exi geant au risque de mettre à jour des divergences profondes, quitte à chercher ensuite - dans un deuxième temps seulement - les com promis exigés aux plans social et légal. La définition suivante, inspirée du Comité Consultatif National d'Ethique en France, est exemplaire à cet égard.

Réflexion approfondie sur les enjeux de la pratique clinique et de la recherche biomédicale afin qu'en ses progrès soit respecté tout homme et tout l'homme ${ }^{14}$.

14 Rédigeant un texte au nom de CCNE en France, Lucien Sève ne parle que d'éthique de la recherche. J'ai élargi l'objet. «Réfléchir de manière approfondie aux enjeux nouveaux de la recherche biologique et 
Curieusement, mais fait intéressant, la fin de cette citation rédigée par le représentant de la famille marxiste au CCNE, reprend ou rejoint une expression présente dans l'encyclique Populorum Progressio publiée par le pape Paul VI en 1967. Si l'on voulait préciser davantage encore la définition, il faudrait expliciter la visée cherchée, nommer les principes de base ou les valeurs fondamentales qui sont en jeu. $\grave{A}$ mon avis, celles-ci concernent ultimement le respect et l'épanouissement de la personne humaine. Cette affirmation implique d'abord le respect et la protection des personnes concrètes: leur liberté, leur autonomie, leur inviolabilité, leur qualité de vie. Elle inclut aussi le respect et la promotion de la vie humaine ellemême et donc du sens de la personne, à moyen et à long terme. Le choix fait par ou pour une personne individuelle peut impliquer une dégradation du sens de la personne. D'où la définition suivante qui met l'accent sur l'aspect objectif ou structurel de la personne humaine.

La bioéthique désigne la recherche de l'ensemble des exigences du respect et de la promotion de la vie humaine ou de la personne dans le champ de la santé ou dans le secteur biomédical ${ }^{15}$.

Remarquons que cette quatrième conception de la bioéthique n'exclut pas les trois précédentes. Au contraire, elle les inclue comme tâches, comme parties intégrantes, mais elle ne s'y limite pas. Ainsi la bioéthique inclut l'analyse de cas, l'analyse de dilemmes, l'aide à la décision; elle est un processus de régulation sociale, mais elle est plus que cela : elle est une réflexion interdisci plinaire à visée éthique. Elle est un projet de réflexion éthique collégiale.

La plupart des définitions de la bioéthique référant aux trois premiers courants inclut une part d'éthique plus ou moins grande,

médicale afin qu'en ses progrès soit respecté tout homme et tout l'homme: telle est la tâche de ce qu'on appelle souvent, d'un terme commode mais discutable, la bioéthique». (CCNE, Recherche biomédicale et respect de la personne humaine, Paris, Doc. française, 1987, p. 9.)

15 Définition personnelle. 
mais celle-ci est souvent minimale. Pour les tenants du dernier courant, seuls, la bioéthique s'avère explicitement une forme d'éthique, une éthique particulière ou mieux un secteur de l'éthique.

Une vision aussi exigeante de la bioéthique peut paraître idéaliste. Elle est loin de faire consensus : le plus souvent, on s'en tient à moins. Elle a cependant sa pertinence et sa nécessité. Certains auteurs croient même que c'est la seule vision valable, critiquant par le fait même toutes les visions réductrices.

\section{2 L'éthique appliquée}

Cette querelle sur la nature de la bioéthique se double ou se pro. longe dans une seconde. Si la bioéthique constitue de l'éthique, peut-on préciser s'il s'agit d'éthique appliquée, une expression popularisée au cours des années soixante dans les pays anglo-saxons? Les uns disent oui, les autres non. À mon avis, cette controverse repose sur une confusion. On ne donne pas au mot appliqué le même sens :

1) Dans un premier sens, le terme appliqué signifie partir des principes pour en déduire une réponse aux cas concrets. Il désigne alors une méthode de réflexion, à savoir une méthode déductive. C'était le sens que donnaient à l'expression les auteurs Beauchamp et Childress dans la lère édition de leur livre en 1979. Et ils affirmaient que la bioéthique est une éthique appliquée et procède par déduction ${ }^{16}$. À l'opposé, David Roy affirme que la bioéthique ne doit pas procéder de manière déductive et donc ne constitue pas de l'éthique appliquée ${ }^{17}$.

2) D'autres donnent au mot le sens de pratique, concret, voire sectoriel, par opposition à théorique, général, fondamental, indépen-

16 T.L. BEAUCHAMP et J.F. CHILDRESS, Principles of Biomedical Ethics. New York/Oxford, Oxford University Press, 1979. Il s'agit d'un livre clé en bioéthique. La plupart des auteurs se situent par rapport à lui, soit en continuité, soit pour le contester. Le livre a bénéficié de quatre éditions : 1979, 1983, 1989, 1994. Dans la dernière édition, les auteurs sont beaucoup plus nuancés sur cette question de méthode.

17 D. ROY et autres, déjà cité. 
damment de la méthode de réflexion (inductive, déductive, etc.) mise en œuvre. On distingue alors l'éthique fondamentale ou générale qui traite des théories éthiques (visée, nature, fondement, struc turation, conditions de possibilité, etc.) des diverses éthiques sectorielles: éthique des affaires, éthique socio-politique, éthique sexuelle, etc. En ce sens, on peut dire que la bioéthique est de l'éthique appliquée, comme le fait la Fédération canadienne des études humaines (1988) ${ }^{18}$.

A mon avis, la bioéthique est effectivement une réflexion d'ordre éthique, orientée vers la pratique, donc une éthique sectorielle. Cependant elle n'est pas liée à une méthode déductive. Au contraire, elle privilégie, d'une certaine façon, l'induction, la casuistique, sans pour autant s'y limiter, sans pour autant exclure la réflexion sur les principes et les fondements.

\section{Controverses sur l'objet}

À propos de l'objet, du contenu ou de l'étendue de la bioéthique, je voudrais exposer trois points de divergences entre les auteurs.

1) J'ai déjà évoqué que, à la suite de Van Rensselaer Potter, certains incluent dans la bioéthique toute la réflexion éthique sur les vivants, donc l'écologie, la surpopulation, la recherche sur l'animal, etc. et, à l'autre extrême, la guerre, la torture, etc. Il me semble qu'on doit plutôt la restreindre au domaine biomédical, déjà très vaste. Je n'y reviens pas.

2) Plus importante me semble la controverse suivante. La bioéthique se réduit-elle, comme plusieurs le proposent, à l'analyse des

18 La Fédération canadienne des études humaines, The Canadian Federation for the Humanities, Towards a Canadian Research Strategy for Applied Ethics, dir. M. MCDONALD, Ottawa, 1988, 114 p. et 231 p. d'annexes (la citation est à la page 9). Voir aussi M.-H. PARIZEAU, "Éthique appliquée " dans M. CANTO-SPERBER (dir.), Dictionnaire d'éthique et de philosophie morale. Paris, PUF, 1996, pp. 534-540, notamment p. 538. F. LACHANCE, interview de Louise Marcil-Lacoste sur le Rapport de la Fédération canadienne dont elle était membre (Forum, 22.1.90). 
dilemmes éthiques, à la résolution des conflits de valeurs suscités par les nouvelles biotechnologies? On laisserait alors de côté tous les actes de la vie quotidienne - et ils sont les plus nombreux - où il n'y a pas de dilemmes éthiques proprement dit, mais où le rapport à l'autre intervient, notamment le rapport médecin-patient, intervenant-famille, chercheur-sujet. $\grave{A}$ mon avis, cela appauvrirait considérablement la réflexion bioéthique et risquerait d'appauvrir tout autant la pratique. Si l'on peut travailler ensemble à résoudre des dilemmes éthiques, plutôt que de les laisser à la seule délibération de l'intervenant perplexe, pourquoi ne pourrions-nous pas travailler ensemble aussi à cerner et à développer les attitudes profondes, le sens des gestes cliniques, les exigences du dialogue professionnel, les requêtes du respect quotidien de la dignité humaine? C'est tout le champ de la vertu, des vertus, qui est ici ouvert ${ }^{19}$. Cela se met mal en lois, en règlements; cela est très questionnant, éventuellement insécurisant. Mais c'est précisément là l'ordre éthique.

3) Une troisième controverse concerne ce qu'on peut appeler les principaux champs de la bioéthique. La bioéthique a commencé aux États-Unis dans le domaine de la recherche et de l'expérimentation sur l'être humain, notamment à la suite des scandales publics provoqués par certaines recherches. C'est ce secteur qui a amené la création de la National Commission dont j'ai parlé précédemment. L'intérêt s'est répandu rapidement au domaine de la pratique clinique, (disons, pour simplifier) à la suite de certains cas cliniques complexes qui ont donné lieu à des débats publics et à des décisions de tribunaux très médiatisés. À mon avis, ce champ est pratiquement plus investigué que le précédent, aujourd'hui.

Dans l'ensemble du Canada, durant les années 80, l'éthique de la recherche n'a guère préoccupé, malgré les directives du CRM et du CRSH. Au Québec, le champ clinique a aussi été privilégié. On parle très souvent de comité de bioéthique, pour désigner des comités qui s'occupent exclusivement d'éthique clinique. Et certains cher-

19 J.F. DRANE, Becomming a Good Doctor. The Place of Virtue and Character in Medical Ethics, Kansas City (MO), Sheed and Ward, 1988, G. VOYER, Qu'est-ce que l'éthique clinique. Bruxelles/Montréal, Artel/Fides (Catalyses), 1996. 
cheurs prétendent que l'éthique de la recherche ne fait pas partie de la bioéthique.

En France, la bioéthique a été rattachée d'abord au domaine de la recherche et de l'expérimentation sur l'être humain; à preuve, la mission du CCNE créé en 1983 et les objectifs des CCPPRB mis sur pied en 1988 dans toutes les régions de la France (loi modifiée en 1990). L'éthique clinique y entre très lentement : voir la directive de l'Assistance publique de Paris en 1984, le développement du Centre d'éthique médicale de Lille. Le Rapport Lenoir en 1991 en recom. mande la diffusion, sous l'égide du CCNE au mandat élargi; il n'a pas été suivi par le législateur.

Enfin, les questions de politiques de la santé, d'allocation des ressources, de santé publique, etc. prennent de plus en plus d'importance. Elles impliquent de toute évidence une dimension éthique. Les auteurs classiques comme Beauchamp et Childress, puis Engelhardt ${ }^{20}$, incluent ce champ en bioéthique. Au Québec, David Roy fait de même ${ }^{21}$, même si ce secteur semble moins développé.

À mon avis, si l'on veut se donner une approche globale cohérente et approfondie de la bioéthique, il y a lieu de réunir ces trois champs principaux parce qu'ils renvoient à une même perspective de santé, quitte à en faire trois champs de spécialisation: l'éthique clinique, l'éthique de la recherche, l'éthique des politiques publiques de santé 22 , mais le champ global demeurerait présent et ouvert. Le

20 T.J. ENGELHARDT, The Foundation of Bioethics, New York, Basic Books, 1986, par exemple p. 85.

21 D. ROY et al., La bioéthique. Ses fondements et ses controverses, déjà cité, pp. 50-63. Je m'inspire étroitement de son livre, quoique avec des nuances. Voir aussi D. ROY, C.-H. RAPIN, M.R. MORISSETTE, "L'éthique clinique: concept et méthode" dans Archives de l'éthique clinique, no 1 . Au chevet du malade. Montréal, Centre de bioéthique, IRC, 1994, pp. 17-37.

22 Cette classification ne fait pas l'unanimité. Certains auteurs distinguent et même opposent éthique clinique et bioéthique (ex.: M. Siegler, E.D. Pellegrino et D.C. Thomasma, P.O. Baron), à mon avis parce qu'ils identifient la bioéthique au courant canonique. Selon mon expérience, plusieurs personnes oeuvrant dans les comités d'éthique de la recherche 
domaine de la santé serait ainsi entièrement couvert et la préoccupation éthique y serait présente, du moins en principe. Cela ne veut pas dire que chaque comité ou chaque instance qui s'occupe de bioéthique doit tout couvrir. Il est légitime d'avoir des objectifs limités, mais la perspective d'ensemble resterait vivante.

\section{Controverses sur les fondements}

Pour faire bref, au risque encore une fois de caricaturer, on peut dire qu'il y $\mathrm{a}$, face à cette question, trois attitudes possibles : le refus, le recours aux théories éthiques, l'effort de réflexion anthropologique.

1) Plusieurs auteurs refusent la pertinence de cette question. La bioéthique est une pragmatique. Il ne peut y avoir consensus sur les questions de fond. La bioéthique est née précisément du constat de ce pluralisme éthique. De toute manière, cette question conduit à une impasse. Contentons-nous de voir la bioéthique comme un processus de régulation sociale et de chercher ensemble les conditions - toujours provisoires et révisables - d'une pratique prudente et vigilante ${ }^{23}$.

2) La deuxième attitude, à l'inverse, trouve important d'expliciter les a priori de chacun. On pense alors aux diverses théories

seraient surprises d'entendre dire qu'elles font de la bioéthique. Enfin, certains intervenants en éthique et gestion hospitalière ressentent un malaise face à leur inclusion en bioéthique. $\grave{A}$ mon avis, cette querelle relève plutôt d'une volonté de chasse-gardée et/ou d'une conception étriquée de la bioéthique.

23 G. HOTTOIS, "Bioéthique : du problème des fondements à la question de la régulation "dans Bioéthique et libre-examen. Bruxelles, Éd. l'Univers, 1988, pp. 101-111. Concrètement, c'est aussi la position de Beauchamp et Childress, même s'ils analysent plusieurs théories éthiques et présentent leur conception comme une théorie. Yvette LAJEUNESSE et Lukas SOSOE parlent à leurs propos de Théorie éthique sans théorie. (Bioéthique et culture démocratique. Montréal, Harmattan, 1996, pp. 105. 127). 
éthiques : déontologisme, utilitarisme, ontologisme, etc. Les auteurs décrivent ces théories, explicitent les conséquences que chacune entraîne sur l'appréciation de l'avortement, les NTR, l'euthanasie, l'allocation des ressources, la recherche sur les gènes, les embryons, etc. On sait bien qu'il n'y aura pas consensus; on trouve quand même important, au plan réflexif, de saisir et aider à saisir les tenants et aboutissants de la pratique ${ }^{24}$. Ce qui est typique de ce courant, c'est qu'on ne prend pas position pour l'une ou l'autre théorie éthique. On reste neutre, comme en dehors. On évite le débat de fond, sauf peutêtre dans les cercles où on exploite la veine ouverte par Jürgen Habermas sur l'éthique de la discussion 25 .

3) Entre ces deux attitudes, certains adoptent une voie moyenne, une voie qui soit à la fois pratique (susceptible d'un large consensus) et théorique (faisant une place à la réflexion substantielle). Les auteurs cherchent ainsi à éviter de réduire la bioéthique à une pure pragmatique, i.e. à une casuistique plus ou moins incohérente, conduisant finalement à un relativisme éthique généralisé. Par ailleurs, ils ne veulent pas s'enliser dans les querelles d'écoles traditionnelles, très théoriques et abstraites. Cette voie est celle de la réflexion sur les concepts de personnes, d'embryon humain, de corps humain, de dignité, etc. On aura reconnu la voie retenue par le CCNE en France, notamment dans son document sur Recherche biomédicale et respect de la personne humaine 26 .

24 Voir ce qui vient d'être dit, note 23, sur Beauchamp et Childress.

25 Attention. Habermas est souvent utilisé pour dégager les règles nécessaires pour qu'une discussion soit éthique. Plus fondamental chez lui - et c'est ce pourquoi je l'évoque ici - est sa présentation (très abstraite et difficile à lire) de la discussion comme fondement de l'éthique.

26 COMITÉ CONSULTATIF NATIONAL D'ÉTHIQUE, Recherche biomédicale et respect de la personne humaine, rédaction Lucien Sève, Paris, La Doc. française, 1987. Pour une présentation critique de cette approche, voir S. PLOURDE, "Incontournable en éthique biomédicale: le concept de personne ", dans Le Supplément 195 (déc. 1995) 29-58; J.-M. THERRIEN, "La notion de personne selon Lucien Sève » dans Philosopher 17 (1995) 263-280. Un court article qui distingue l'approche philosophique d'un complément théologique à la notion de personne: X. THÉVENOT, La 
La première attitude me semble manquer de dynamisme. La deuxième me semble oublier les préoccupations qui ont donné naissance à la bioéthique: recherche d'une sagesse, recherche d'une réconciliation entre les sciences et les humanités, souci de la décision et de l'action. Seule, la troisième me semble vraiment stimulante, porteuse d'humanité et prometteuse d'avenir.

\section{Malentendus sur les acteurs}

J'aimerais aborder ici très succinctement une question personnalisée : qui est bioéthicien? qui est éthicien? qu'est-ce qu'un expert en éthique? Ici encore distinguons divers aspects de la question.

1) D'abord, quel est le sujet de l'éthique, l'agent moral, et qu'on peut appeler d'une manière très générale éthicien? Pas besoin de chercher des grands noms, des prix Nobel; la réponse est bien plus simple. C'est chacun de nous. C'est chacun de ceux qui œuvrent dans le monde de la santé. De même que l'éthique ou la morale, en général, n'est pas réservée aux experts, mais concerne chaque sujet humain, interpelle chaque personne humaine, de même la bioéthique concerne tous les intervenants du monde de la santé (professionnels, administrateurs, patients, proches, etc.). Là où il y a décision à prendre, réflexion à faire, liberté à engager, il y a éthique et - dans le champ de la santé - bioéthique ${ }^{27}$.

Plusieurs font de la bioéthique sans le savoir. Mieux vaut en être conscient. Il est possible alors d'être plus attentif, plus critique. Il est possible de se donner une formation supplémentaire pour être capable

bioéthique, Paris, Centurion, 1989, chapitre 3: «La personne et ses limites », pp. 53-63.

27 "Or l'éthique est essentiellement liée à l'action et relève donc primordialement de la raison pratique, non de la raison théorique, et la raison pratique, en tant que composante fondamentale de la raison, appar. tient par essence à tout homme, " J. LADRIÈRE, "Éthique et tâche de la pensée. L'éthique et la raison pratique " dans L'Éthique : interprétations et positionnements multiples. Bruxelles, Éditions ECCE a.s.b.l. (Forum éthique), Actes du 19 juin 1993, p. 1. 
de mieux analyser les questions, discerner les enjeux, suggérer des éléments de solution, développer des attitudes adéquates.

Quant au titre de bioéthicien, d'expert en bioéthique ou de conseiller en éthique, il présuppose une formation spécifique adéquate. Les balises sont difficiles à fixer. Il me semble que le titre est parfois usurpé.

2) Toujours au sujet des acteurs, la bioéthique n'est pas réservée à ceux qui font partie d'un comité de bioéthique. Ces comités spécialisés sont importants. Loin de moi l'idée de minimiser leur rôle, mais la bioéthique n'est pas leur chasse gardée. On peut faire de la bioéthique à son bureau, on en fait sur les étages de manière informelle avec d'autres intervenants. On en fait dans certains comités, commissions, groupes qui n'ont pas ce nom.

On devrait en faire spécialement dans les comités spécifiques, mais cela n'est pas toujours facile, ni évident. Il reste difficile de distinguer la normativité éthique des normativités administrative, éco nomique, légale et professionnelle ${ }^{28}$.

3) Le titre de conseiller ou d'expert en bioéthique prête à confusion. Certains le contestent, voire le ridiculisent ${ }^{29}$. Si on le conçoit, le présente et le vit comme celui qui détient la vérité, celui qui

28 L. BÉGIN, "La normativité dans les comités d'éthique clinique " dans Hôpital et éthique, déjà cité, pp. $32-57$ (citation p. 34). Voir aussi É. GAGNON, " Usage du droit et usage de la parole : normes juridique, professionnelle et éthique » dans Hôpital et éthique, pp. 199-219; G. ROCHER, «La bioéthique comme processus de régulation sociale : le point de vue de la sociologie » dans J.-H. PARIZEAU, Bioéthique, méthodes et fondements. Montréal, ACFAS, 1989, pp. 49-62; G. ROCHER, "Y a-t-il des normes d'allocation des équipements coûteux en milieu hospitalier? » dans Revue de Droit de l'Université de Sherbrooke (février 1990) 221-229; M.-L. DELFOSSE, «Les comités de la recherche : l'éthique et le droit à l'épreuve du principe de justice " (Conférence, congrès de Montréal en 1996).

29 A.L. CAPLAN, "Moral Experts and Moral Expertise" dans B. HOFFMASTER, B. FREEDMAN, G. FRASER (dir.), Clinical Ethics. Theory and practice. Clifton, Human Press, 1989, p. 67 etc.; Y. LAJEUNESSE et L. SOSOE, Bioéthique et culture démocratique, pp. 209-230. 
donne la bonne solution que le demandeur n'aurait qu'à suivre, la critique est fondée. Cette conception déresponsabilise le sujet en dévalorisant la conscience personnelle (de l'intervenant ou du sujet) et en niant la responsabilité professionnelle (dans le cas du professionnel). Elle attribue par ailleurs au bioéthicien une autorité et un pouvoir qui ne lui reviennent pas. Elle dénature enfin le sens de l'éthique.

À mon avis, l'expertise éthique (mais le mot est sujet à malentendu) est d'un autre ordre. Il ne s'agit pas de décider à la place d'un autre. Il s'agit, grâce à un certain savoir théorique, grâce à une connaissance de cas similaires, grâce à une familiarisation avec le monde de la santé, grâce à une expérience acquise, d'aider l'autre ou les autres à faire une analyse éthique et à porter un jugement éthique.

Pour expliciter cette affirmation, on peut distinguer diverses tâches :

- aider à cerner les faits pertinents, à dégager les valeurs, les principes et les règles en jeu, à être attentif à tous les aspects, à ouvrir et expliciter des pistes de solution;

- fournir, le cas échéant, certaines explications, comme d'expliquer un concept, une expression éthique, de situer une notion dans un ensemble, de signaler la faiblesse ou l'inadéquation d'un argument;

- sensibiliser à l'importance du singulier et de l'universel, au souci des personnes concrètes et du sens de l'humain;

- attirer l'attention éventuellement sur les attitudes, les dispositions intérieures, la manière d'agir ${ }^{30}$.

30 Voir J. LADRIÈRE, "Note sur l'institutionnalisation de l'éthique » dans L'éthique: interprétations et positionnements multiples, pp. 11-14. Paradoxalement, malgré la dure critique faite à l'expertise bioéthique, Lajeunesse et Sosoe reconnaissent la pertinence d'un spécialiste de l'éthique (p. 217, 223), dont l'apport correspond sensiblement avec le rôle que je décris ici. Il y a donc pour une bonne part ambiguité sur le mot. 
Bref, l'éthicien ou le bioéthicien ne vient pas éliminer ou faire ombrage aux autres acteurs. Au contraire. Il vient aider chacun à prendre ses responsabilités au plan éthique.

\section{Malentendus sur les comités}

1) Quand on parle de la composition des comités de bioéthique, on réfère souvent à deux règles: qu'il soit multidisciplinaire, pour assurer la richesse des analyses; qu'il comporte des personnes extérieures à l'établissement, à la fois pour éviter une sorte d'enfermement de la pensée et par souci démocratique. Cela n'est pas toujours facile à mettre en pratique. Et on présente parfois des sophismes pour y échapper.

2) L'objectif général des comités est de réfléchir sur les enjeux éthiques de la pratique, entendu dans un sens large. Ou mieux d'aider à tenir compte de la dimension éthique de toute décision. Il vaut la peine de préciser que l'avis d'un comité ne cherche pas à se substituer à la conscience d'un sujet, ni à la responsabilité professionnelle d'un intervenant, comme certains le craignent; mais d'aider à analyser une situation, éventuellement d'aider le demandeur perplexe à prendre sa décision. Ce n'est pas à tort que l'on insiste un peu partout sur la nature facultative de ces avis et l'importance pour le comité de se centrer sur, sinon de se limiter à la dimension éthique. Il n'y a donc pas lieu d'être déçu si un demandeur ne suit pas un avis.

3) Si le rôle des comités est de réfléchir sur les enjeux de la pratique médicale, biomédicale, paramédicale, il ne doit pas se cantonner dans l'éthique clinique et l'éthique de la recherche, en excluant l'éthique des politiques de santé, des choix administratifs, etc. J'ai parlé précédemment des trois grands secteurs de la bioéthique. Mais à cet effet, il faut peut-être penser à établir un comité différent du $\mathrm{CEC}$ et du CER, comité qu'on pourrait appeler d'éthique institutionnelle (CEI). Ce n'est pas forcément les mêmes personnes qui ont l'intérêt et les qualifications pour réfléchir à ces questions macro-

Sans nier la passion des auteurs en faveur de la responsabilisation des sujets. 
éthiques. Les enjeux éthiques y sont pourtant majeurs, quoique rarement explicités, transparents.

4) Un quatrième malentendu porte sur le bénévolat. Pour participer à un comité de bioéthique, il faut, entre autres, avoir le goût et le temps. Cela demande de l'intérêt et de la disponibilité. Cependant, si on veut que ces comités fassent vraiment œuvre efficace, il faut être convaincu de deux choses:

- la première, qu'il y ait un ou deux membres possédant une formation spécifique en éthique ou bioéthique et qui puissent agir comme personnes-ressources, soit un philosophe ou un théologien qui s'est intéressé aux milieux de la santé, soit un professionnel de la santé qui s'est donné une formation complémentaire en éthique.

- le second élément important est que l'une de ces personnes puisse y mettre vraiment du temps : temps pour la recherche, temps pour l'écriture, temps pour tenir les dossiers, temps pour les contacts, temps pour assurer le suivi, etc. Des comités d'éthique clinique prennent $2-3$ ans pour établir des lignes directrices sur un thème: c'est trop long, c'est démotivant, démobilisant. Des CER analysent $10-12$ protocoles de recherche par mois : une préparation adéquate demande 3-4 heures par protocole. Pour un travail sérieux, il est impossible de compter sur le seul bénévolat. À mon avis, il est essentiel de dégager vraiment quelqu'un de sa tâche s'il est déjà employé de l'établissement ou d'engager spécifiquement quelqu'un de l'extérieur, en le rémunérant adéquatement. Un malentendu tenace consiste à penser que travail éthique et rémunération sont incompatibles. Pour pallier des abus éventuels, diverses formules sont possibles ${ }^{31}$.

31 Au Canada, un récent document des trois Conseils subventionnaires demande que les comités d'éthique de la recherche (CER) "disposent d'une indépendance financière et administrative suffisante pour remplir leurs obligations». Quoique vague, la formule peut très bien inclure la rémunération d'une personne formée en éthique. (Voir CRM, CRSNG, CRSH, Code d'éthique de la recherche avec des êtres humains. Ottawa, juillet 1997, 2e partie, chap. 2 B, no 2). Au Québec, Le plan d'ac- 
Ce changement serait d'ailleurs cohérent avec l'effort que fait la société, via l'Université, pour former des diplômés.

5) Le dernier point concerne le consensus. Ce concept, en effet, est source lui aussi de malentendus. Le premier porte sur le sens du mot. Celui-ci en effet peut désigner deux choses : tantôt un processus de décision (on parlera ainsi de procéder par consensus plutôt que par vote), tantôt le résultat obtenu (on dira alors qu'on a fait consensus ou non) $)^{32}$.

Je ne parlerai pas du processus de discussion, lequel est correct quand on a respecté, de manière explicite ou non, certaines règles que les psychologues et les logiciens ont aidé à expliciter.

Je voudrais attirer l'attention sur le consensus-résultat, et notamment sur le culte parfois exagéré de l'unanimité. Dans certains comités, on valorise tellement le consensus qu'on arrive à faire de faux compromis sous couvert d'unanimité ${ }^{33}$, qu'on remette à plus tard une décision qui a une certaine urgence, ou qu'on sorte déçu et déstabilisé de la rencontre. Certes, l'unanimité, la vraie, est désirable : elle est source de satisfaction, elle est sécurisante. Sans donner une certitude absolue, elle correspond à la meilleure approximation de la vérité à laquelle on peut arriver. Pourtant on ne peut pas la forcer : le pluralisme axiologique est parfois insurmontable; l'éthique

tion ministériel en éthique de la recherche et en intégrité scientifique contient une indication analogue (Québec, MSSSS, été 98).

32 L. BÉGIN, «L'éthique par consensus » dans M.H. PARIZEAU (dir.), Hôpital et Éthique, pp. 176-189. Voir aussi les références de la note 27.

33 Le vrai consensus désigne donc "l'accord intervenu entre des personnes pouvant - et ayant de fait - participer activement à la discussion en toute liberté d'expression et d'opinion" (L. BÉGIN, "L'éthique par consensus ", p. 183). Ou encore, de manière plus développée : "un accord explicite et critique intervenant après analyse de la situation problématique et discussion argumentée entre les personnes effectivement concernées par la décision et ses conséquences" (J.-F. MALHERBE, L. ROCCHETTI ET A.-M. ROIRE-LAVIGNE, "Validité et limites du consensus en éthique clinique», p. 532. 
est elle-même le domaine de l'incertitude. Il n'y a pas de honte ou d'échec à ne pas toujours y arriver. Soit dit, sans dévaloriser tous les efforts mis en œuvre pour y arriver ${ }^{34}$.

\section{Conclusion}

Deux mots de conclusion pour résumer l'essentiel de mon propos. La bioéthique désigne des pratiques et des discours ayant pour objet de clarifier et d'expliciter les questions à portée éthique soulevées par le développement des technologies dans le monde de la santé et de la vie humaine ou, encore, des réflexions ayant pour objet d'expliciter la dimension éthique des décisions à prendre concernant la santé, la maladie, la vie, la mort... par moi, l'autre, les autres. Elle a une visée explicitement éthique.

Or tout ce champ de pratique et de réflexion est le lieu de controverses inévitables, mais aussi d'ambiguités et de malentendus dommageables. La grandeur de la bioéthique risque d'être minée par ces misères. Ceux qui croient à sa grandeur doivent être habités par un souci constant de clarté et de clarification. Cette citation du CCNE en France l'explicite admirablement.

Le vocabulaire scientifique tend à la rigueur. Le vocabulaire juridique aussi. Trop souvent, semble-t-il, on paraît admettre qu'un effort analogue ne serait pas exigible du vocabulaire éthique.

Entre une science des plus précises et une éthique un peu trop floue, comment s'étonner si la partie est inégale? Sans doute n'obtiendra-t-on jamais et n'y aurait-il pas même de sens à réclamer que les termes de la seconde, signes d'une expérience d'un tout autre ordre que celle du laboratoire et enjeux de controverses essentiellement inépuisables, puissent être rigoureux selon les critères de la première. Si c'était possible, cela risquerait d'ailleurs d'instituer une bioéthique au savant jargon fâcheuse -

34 Très belle analyse de l'éthique consensuelle, à partir des travaux du CCNE en France, par N. AUMONIER, "Les limites de l'éthique consensuelle " dans Communio 18/5 (sept.-oct. 93) 99-126. 
ment coupée du parler courant de la morale. Mais prévenir le malentendu désastreux, débusquer l'expression fallacieuse, affiner le concept indispensable fait éminemment partie du possible et du nécessaire ${ }^{35}$.

35 CCNE, Recherche biomédicale et respect de la personne humaine, p. 24. 